\title{
Interpage and interpixel cross talk in orthogonal (wavelength-multiplexed) holograms
}

\author{
Amnon Yariv \\ California Institute of Technology, Pasadena, California 91125
}

Received October 16, 1992

\begin{abstract}
The problem of cross talk in image-bearing wavelength-multiplexed holograms was raised recently [A. Yariv, in Digest of Conference on Nonlinear Optics (Optical Society of America, Washington, D.C., 1992), postdeadline paper E-2]. In the limit of a large aperture (lens, crystal) it is shown that the cross talk is independent of the information content. The reduction of the hologram volume is shown to introduce interpixel as well as interpage cross talk.
\end{abstract}

A recently proposed method of storing data in multiple holograms ${ }^{1-3}$ is based on counterpropagating waves and wavelength multiplexing. The fundamental feature of this method is that the presence of information causes the grating $\mathbf{k}$ vectors to spread uniformly with minimal crowding (in $\mathbf{k}$ space) so that to a high degree of approximation the cross talk is independent of the information content-a feature not shared by the conventional method, which employs angular multiplexing. In this paper I analyze the problem of interpixel cross talk in this (counterpropagating waves and wavelength multiplexing) format.

Figure 1 shows the recording geometry. A page $s$ recorded with wavelength $\lambda_{s}$ contains $N^{2}$ pixels. When a $z$-directed plane wave at $\lambda_{s}$ is incident upon the transparency, each pixel gives rise to a bundle of plane waves at the recording crystal, which is placed a focal distance $f$ to the right of the lens (in the Fourier plane). The complex amplitude of the field at the crystal that is due to page $s$ can be expressed as

$$
\begin{gathered}
E^{(s)}=\sum_{m=1}^{N^{2}} \int_{-\infty}^{\infty} A_{m}^{(s)}\left(k_{\perp}^{\prime \prime}\right) \exp \left[-i \mathbf{k}_{\perp}^{\prime \prime} \cdot \mathbf{r}_{\perp}-i \mathbf{k}_{z}^{\prime \prime(s)} z\right] \mathrm{d}^{2} k_{\perp}^{\prime \prime}, \\
k_{z}^{\prime \prime(s)}=\sqrt{k_{s}^{2}-\left(k_{\perp}^{\prime \prime}\right)^{2}}, \quad k_{s}=\frac{\omega_{s}}{c} n_{s}
\end{gathered}
$$

and $m$ is a shorthand notation for the two integers $m_{x}$ and $m_{y}$ that specify the address of the pixel. If the original pixels are squares with a size $\delta$, we can use basic Fourier optics to obtain the spatial frequency spectrum in the focal plane of pixel $m$ of page $s$,

$$
\begin{aligned}
A_{m}^{(s)}\left(k_{\perp}\right)= & A_{\mathrm{mo}}^{(s)} \prod\left(\frac{-k_{x}-m_{x} S \frac{k_{s}}{f}}{\delta k_{s} / f}\right) \\
& \times \prod\left(\frac{-k_{y}-m_{y} S \frac{k_{s}}{f}}{\delta k_{s} / f}\right),
\end{aligned}
$$

where $S$ is the period (in $x$ or $y$ ) of the pixel array and $\Pi$ is the rectangular function

$$
\prod(x)= \begin{cases}1 & |x| \leq 1 / 2 \\ 0 & \text { otherwise }\end{cases}
$$

The spatial frequency spectrum of each bundle at the crystal is thus uniform and limited to an interval $\Delta k_{x y}=\delta k / f$. Moreover the spectrum of any pixel is zero at the $\left(\mathbf{k}_{\perp}\right)$ domain occupied by any other pixel, as shown in Fig. 2.

The hologram $s$ is written by the interference of the field $E^{(s)}$ and the oppositely traveling plane wave $E_{s} \exp \left(i k_{s} z\right)$ of the same frequency. The resulting hologram involves the product $E_{s}{ }^{*} \exp \left(-i k_{s} z\right) E^{(s)}$. A total of $h$ equal-amplitude holograms, each with a unique wavelength, are recorded each with (two) incident waves along $z$ and $-z$. The total index-ofrefraction perturbation (i.e., the hologram) can be written as

$$
\begin{aligned}
\Delta n(\mathbf{r})= & \sum_{s=1}^{h} \sum_{m=1}^{N^{2}} E_{R}^{*(s)} \kappa \int A_{m}^{(s)}\left(\mathbf{k}_{\perp}{ }^{\prime \prime}\right) \\
& \times \exp \left\{-i \mathbf{k}_{\perp}^{\prime \prime} \cdot \mathbf{r}_{\perp}-i\left[k_{z}^{\prime \prime(s)}+k_{s}\right] z\right\} \mathrm{d}^{2} \mathbf{k}_{\perp}^{\prime \prime}+\text { c.c. }
\end{aligned}
$$

where $\kappa$ is a constant denoting the recording efficiency, which is taken to be the same for all holograms.

To retrieve, say, page $(\ell)$ we illuminate the hologram with a plane wave $E_{\text {read }}^{\ell} \exp \left(i k_{\ell} z\right)$ at $\lambda_{\ell}$ incident along the same direction as the recording reference wave at $\lambda_{\ell}$. We can expand the resulting diffracted wave as

$E_{d}^{(\ell)}=\sum_{t} \int a_{t}^{(\ell)}\left(\mathbf{k}_{\perp}^{\prime}\right) \exp \left[-i \mathbf{k}_{\perp}^{\prime} \cdot \mathbf{r}_{\perp}-i \mathbf{k}_{z}^{(\ell)} z\right] \mathrm{d}^{2} \mathbf{k}_{\perp}^{\prime}$,

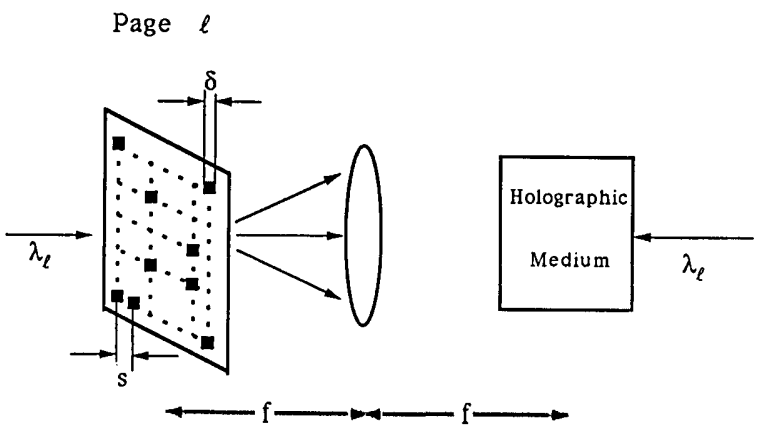

Fig. 1. Geometry used to record a single page $(\ell)$ at $\lambda_{\ell}$. The depth of focus of the lens is much larger than the crystal length. 


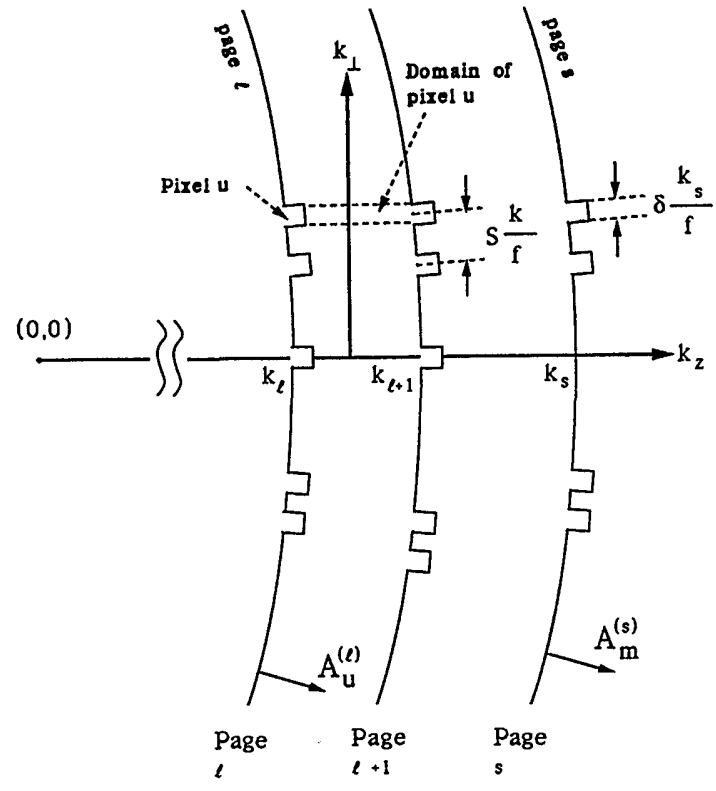

Fig. 2. Plot in one dimension of the spatial frequency $\left(\mathbf{k}_{1}\right)$ dependence of the three field amplitudes of the recorded (and reconstructed) pages $(\ell, \ell+1$, and $s)$ in the focal plane in the limit of negligible transverse aperturing (i.e., large lens, large crystal cross-sectional area).

where $a_{t}^{(\ell)}\left(\mathbf{k}_{\perp}^{\prime}\right)$ represents a bundle of plane waves centered on the nominal direction $\mathbf{k}_{t \perp}^{(\ell)}$. For a perfect reproduction of the page $\ell$ we will need to show that

$$
a_{t}^{\ell}\left(\mathbf{k}_{\perp}\right)=\text { const. } A_{t}^{(\ell)}\left(\mathbf{k}_{\perp}\right)
$$

with the same constant for all $t$, i.e., that there is a one-to-one correspondence between each pixel of the incident wave and the corresponding diffracted wave. The expansion as in Eqs. (1) and (5) in plane-wave bundles is a key feature of our formalism. Our next task is to solve for $a_{t}^{(\ell)}\left(\mathbf{k}_{\perp}\right)$ and to see if and under what conditions relation (6) is satisfied. We start with the wave equation for the total reconstructed field at $\lambda_{\ell}$ :

$$
\nabla^{2} E_{\ell}+\omega^{2} \mu \epsilon_{0}\left[n_{\ell}^{2}+n_{\ell} \Delta n(\mathbf{r})\right] E_{\ell}=0,
$$

where

$$
\begin{aligned}
E_{\ell}= & E_{\text {read }}^{\ell} \exp \left(i k_{\ell} z\right) \\
& +\sum_{t} \int a_{t}^{(\ell)}\left(\mathbf{k}_{\perp}^{\prime}\right) \exp \left[-i \mathbf{k}_{\perp}^{\prime} \cdot \mathbf{r}_{\perp}-i \mathbf{k}_{z}^{(\ell)} z\right] \mathrm{d}^{2} \mathbf{k}_{\perp}^{\prime} .
\end{aligned}
$$

Use of Eq. (4) and neglecting second derivatives $\partial^{2} a_{t} / \partial z^{2}$ leads to the paraxial wave equation

$$
\begin{gathered}
2 i \sum_{t} \int k_{t z}^{(\ell)}\left[\frac{\mathrm{d}}{\mathrm{d} z} a_{t}^{(\ell)}\left(\mathbf{k}_{\perp}^{\prime}\right)\right] \exp \left[-i \mathbf{k}_{\perp}^{\prime} \cdot \mathbf{r}_{\perp}-i \mathbf{k}_{z}^{(\ell)} z\right] \mathrm{d}^{2} \mathbf{k}_{\perp}^{\prime} \\
=\sum_{s}^{h} \sum_{m} \beta \int A_{m}^{(s)}\left(\mathbf{k}_{\perp}^{\prime \prime}\right) \\
\times \exp \left\{-i\left[\left(k_{z}^{\prime(s)}+k_{s}-k_{\ell}\right) z+k_{\perp}^{\prime \prime} \cdot \mathbf{r}_{\perp}\right]\right\} \mathrm{d}^{2} \mathbf{k}_{\perp}^{\prime \prime}, \\
k_{z}^{\prime(s)}=\sqrt{{k_{s}}^{2}-k_{\perp}^{\prime \prime 2}},
\end{gathered}
$$

where $\beta$ is a constant resulting from combining a number of factors. We multiply both sides of Eq. (8) by $\exp \left(i \mathbf{k}_{\perp} \cdot \mathbf{r}_{\perp}\right)$ and integrate over the infinite transverse plane. The result is

$$
\begin{aligned}
\sum_{t} 2 i k_{t z}^{(\ell)} \frac{\mathrm{d}}{\mathrm{d} z} a_{t}^{(\ell)}\left(\mathbf{k}_{\perp}\right) \exp \left(-i \sqrt{k_{\ell}{ }^{2}-{k_{\perp}}^{2}} z\right) \\
=\sum_{s} \sum_{m} \beta A_{m}^{(s)}\left(\mathbf{k}_{\perp}\right) \exp \left[-i\left(\sqrt{{k_{s}}^{2}-{k_{\perp}}^{2}}-k_{\ell}+k_{s}\right) z\right],
\end{aligned}
$$

where we used the relation

$$
\begin{aligned}
\int_{-\infty}^{\infty} \exp \left[i\left(\mathbf{k}_{\perp}-\mathbf{k}_{\perp}^{\prime \prime}\right) \cdot \mathbf{r}_{\perp}\right] \mathrm{d}^{2} \mathbf{r}_{\perp} & \\
= & 4 \pi^{2} \delta\left(\mathbf{k}_{x}-\mathbf{k}_{x}^{\prime \prime}\right) \delta\left(\mathbf{k}_{y}-\mathbf{k}_{y}^{\prime \prime}\right) .
\end{aligned}
$$

Since we chose, without loss of generality, $\mathbf{k}_{\perp}$ to lie in the domain of pixel $u$, it follows from Eq. (2) that $A_{m}^{(s)}\left(\mathbf{k}_{\perp}\right)=0$ unless $m=u$. (This can also be seen from Fig. 2.) In other words, there is no overlap between the spatial frequency spectra corresponding to different pixels of a given page. We thus need to keep only the $m=u$ term on the right-hand side of Eq. (9), which then becomes

$$
\begin{aligned}
& \sum_{t} 2 i k_{t z}^{(\ell)} \frac{\mathrm{d}}{\mathrm{d} z} a_{t}^{(\ell)}\left(\mathbf{k}_{\perp}\right) \exp \left(-i \sqrt{k_{\ell}^{2}-k_{\perp}^{2}} z\right) \\
& =\sum_{s} \beta A_{u}^{(s)}\left(\mathbf{k}_{\perp}\right) \exp \left[-i\left(\sqrt{{k_{s}^{2}}^{2}-k_{\perp}{ }^{2}}-k_{\ell}+k_{s}\right) z\right] .
\end{aligned}
$$

Equation (10) is not quite satisfactory because of the summation on the left-hand side. Let us consider first the case in which only one hologram (page), say $\ell$, is recorded. The summation over $s$ reduces in this case to the $s=\ell$ term. Let us claim, and later justify, that we need keep but the single term $t=u$ in Eq. (10). The result is

$$
2 i k_{u z}^{(\ell)} \frac{\mathrm{d}}{\mathrm{d} z} a_{u}^{(\ell)}\left(\mathbf{k}_{\perp}\right)=\beta A_{u}^{(\ell)}\left(\mathbf{k}_{\perp}\right)
$$

so that

$$
a_{u}^{(\ell)}\left(\mathbf{k}_{\perp}\right)=g A_{u}^{(\ell)}\left(\mathbf{k}_{\perp}\right) z \quad(g \text { is a constant }) .
$$

Thus, as is the case for $A_{t}^{(\ell)}\left(\mathbf{k}_{\perp}\right), a_{t}^{(\ell)}\left(\mathbf{k}_{\perp}\right)$ is zero unless $t=u$. This justifies keeping only the term $t=u$ in Eq. (10) and renders Eq. (12) self-consistent. Using Eq. (12) in Eqs. (1) and (5) leads to $E_{d}^{(\ell)}=g z E^{(\ell)}$, i.e., perfect reconstruction of page $\ell$. The consequence of Eq. (12) is that there exists no interpixel cross talk in a given hologram. (As is shown below, this is strictly true for infinite cross sections.) If we now return to the multipage hologram, Eq. (10) becomes

$$
\begin{aligned}
& 2 i k_{u z}^{(\ell)} \frac{\mathrm{d}}{\mathrm{d} z} a_{u}^{(\ell)}\left(\mathbf{k}_{\perp}\right)=\beta\left\{A_{u}^{(\ell)}\left(\mathbf{k}_{\perp}\right)\right. \\
& \left.+\sum_{s \neq \ell} \exp \left[-i 2\left(k_{s}-k_{\ell}\right)\left(1+\frac{k_{\perp}{ }^{2}}{4 k_{\ell} k_{s}}\right) z\right] A_{u}^{(s)}\left(\mathbf{k}_{\perp}\right)\right\},
\end{aligned}
$$

where for the sake of simplicity we have assumed that $\left|\mathbf{k}_{\perp}\right|^{2} \ll k_{\ell}{ }^{2}, k_{s}{ }^{2}$ in the exponent (carrying the exact 
expression has no impact on any of our conclusions). The solution of Eq. (13) is

$$
\begin{aligned}
a_{u}^{(\ell)}\left[\mathbf{k}_{\perp}\right] & =g A_{u}^{(\ell)}\left(\mathbf{k}_{\perp}\right) z \\
& +\sum_{s \neq \ell}^{h} g z \frac{\sin \left(k_{s}-k_{\ell}\right)\left(1+\frac{k_{\perp}{ }^{2}}{4 k_{\ell} k_{s}}\right) z}{\left(k_{s}-k_{\ell}\right) z} A_{u}^{(s)}\left(\mathbf{k}_{\perp}\right),
\end{aligned}
$$

where we recall that we chose, without loss of generality, $\mathbf{k}_{\perp}$ to lie within the domain (see Fig. 2) of pixel $u$. The first term on the right-hand side of Eq. (14) represents the Bragg-matched ideal contribution to pixel $u$ of page $\ell$ of the diffracted field from the same pixel in the recorded hologram. The summation accounts for the coherent cross-talk contributions from the same pixel in all the remaining $h-1$ recorded holograms.

Consider, for a moment, the case of recording $h$ holograms bearing no pictorial information, i.e., plane-wave holograms. This is a special case of Eq. (14) with only one pixel, say $u$. Equation (14), which involves a summation over pages and not pixels, remains unchanged. It follows that the cross talk in wavelength-multiplexed counterpropagation holograms (called orthogonal data storage in Ref. 4) is independent of the amount of stored information, as claimed. ${ }^{4}$

In the above I have taken the transverse dimensions of the lens, or crystal, as infinite. The formalism, however, can readily account for the effects of transverse truncation. Let us assume that the crystal causes a rectangular aperturing with an effective aperture $D \times D$, where $D$ is the transverse dimension of the crystal. [Hard truncation results when $D<(2 \lambda / \delta) f$.] If the lens diameter is smaller than $D$, we use it (the lens diameter) instead of $D$. Straightforward Fourier/Fresnel optics leads to an expression for the spatial frequency $\left(\mathbf{k}_{\perp}\right)$ spectrum of a given pixel field, say $(s, m)$, at the focal plane:

$$
\begin{aligned}
A_{m}^{(s)}\left(\mathbf{k}_{\perp}\right)= & A_{\mathrm{mo}}^{(s)}\left(\frac{2 \pi \delta D}{\lambda f}\right)^{2} \operatorname{sinc}\left[\left(-k_{y}-m_{y} S \frac{k_{s}}{f}\right) \frac{D}{2}\right] \\
& \times \operatorname{sinc}\left[\left(-k_{x}-m_{x} S \frac{k_{s}}{f}\right) \frac{D}{2}\right] .
\end{aligned}
$$

This contrasts with the band-limited spectrum of the infinite cross-section case [Eq. (2)]. The prefactor $(2 \pi \delta D / \lambda f)^{2}$, which, in the case of strong truncation, is small compared with unity, accounts for the reduction of diffracted power owing to the smaller (truncated) interaction volume. More importantly, the individual pixel spectra in this case are broader $\left(\Delta \mathbf{k}_{\perp} \sim\right.$ $\pi / D)$ than in the infinite-aperture case of Eq. (2) $\left(\Delta \mathbf{k}_{\perp}=2 \pi \delta / \lambda f\right)$ and, because of the sinc dependence, invade each other's spectral domain. The result is that the detection of the energy of a given pixel involves inevitable contributions from other pixels, i.e., cross talk. This can be seen if we use Eq. (15) to solve, as in Eq. (14), for the spectrum of the diffracted field of pixel $u$ during the reconstruction of page $\ell$. The result is

$$
\begin{aligned}
& a_{u}^{(\ell)}\left(\mathbf{k}_{\perp}\right) \cong\left(\frac{2 \pi \delta D}{\lambda f}\right)^{2} g z A_{u}^{(\ell)}\left(\mathbf{k}_{\perp}\right)+\sum_{m \neq u}\left(\frac{2 \pi \delta D}{\lambda f}\right)^{2} g z A_{\mathrm{mo}}^{(\ell)} \\
& \times \operatorname{sinc}\left[\left(-k_{x}-m_{x} S \frac{k_{s}}{f}\right) \frac{D}{2}\right] \operatorname{sinc}\left[\left(-k_{y}-m_{y} S \frac{k_{s}}{f}\right) \frac{D}{2}\right] \\
& +\sum_{s \neq \ell} \sum_{m}\left(\frac{2 \pi \delta D}{\lambda f}\right)^{2} g z A_{\mathrm{mo}}^{(s)} \operatorname{sinc}\left[\left(-k_{x}-m_{x} S \frac{k_{s}}{f}\right) \frac{D}{2}\right] \\
& \times \operatorname{sinc}\left[\left(-k_{y}-m_{y} S \frac{k_{s}}{f}\right) \frac{D}{2}\right] \\
& \times \operatorname{sinc}\left[\left(k_{x}-k_{\ell}\right)\left(1+\frac{k_{\perp}{ }^{2}}{4 k_{\ell} k_{s}}\right) z\right],
\end{aligned}
$$

where $\mathbf{k}_{\perp}=\hat{x} k_{x}+\hat{y} k_{y}$. The second term on the righthand side of relation (16) represents cross talk into pixel $u$ of page $\ell$ from all other pixels on the same page. The double summation on the right-hand side gives the cross talk from all pixels $(m \neq u$ as well as $m=u$ ) in all other pages. This, truncation-induced cross talk can be reduced below the minimum infiniteaperture level given by Eq. (14) by increasing the interpixel separation $S$.

An immediate consequence of relation (16) is the broadening of the pixel spectra $\operatorname{sinc}\left\{\left[-k_{x}-\right.\right.$ $\left.\left.m_{x} S\left(k_{s} / f\right)\right](D / 2)\right\}$ function, which increases as the crystal cross-section diameter $D$ decreases. This implies that, all other factors remaining the same, the cross talk in holograms using fibers with a diameter of, say, $1 \mathrm{~mm}$ will be 100 times larger than the cross talk in a crystal with a diameter of $1 \mathrm{~cm}$.

The author acknowledges with pleasure numerous crucial discussions with L. Glasser, R. Guenther, V. Leyva, G. Rakuljic, B. Crosignani, and M. Segev. This research was supported by the U.S. Army Research Office, the U.S. Air Force Office of Scientific Research, and the Defense Advanced Projects Agency.

\section{References}

1. G. Rakuljic, A. Yariv, and V. Leyva, in Digest of Conference on Photorefractive Materials, Effects, and Devices (Optical Society of America, Washington, D.C., 1991), paper MD-3.

2. G. Rakuljic, V. Leyva, and A. Yariv, Opt. Lett. 17, 1471 (1992).

3. V. Leyva, G. Rakuljic, and A. Yariv, in Annual Meeting, Vol. 17 of 1991 OSA Technical Digest Series (Optical Society of America, Washington, D.C., 1991), paper FU-7.

4. This material was first presented by the author in Digest of Conference on Nonlinear Optics (Optical Society of America, Washington, D.C., 1992), postdeadline paper E-2. 